\title{
An Adaptive Structures Electro-Mechanical Device for Dynamic Flow Control Applications
}

\author{
Russell F. Osborn ${ }^{*}$, Sridhar Kota ${ }^{\dagger}$ and Joel Hetrick ${ }^{\ddagger}$ \\ FlexSys Inc., 2000 Hogback Rd., Suite 9, Ann Arbor, MI 48105 \\ Donald Geister $^{\S}$ \\ University of Michigan, Ann Arbor, MI \\ Carl Tilmann ${ }^{\mathbb{I}}$ \\ AFRL Air Vehicle Directorate \\ Wright-Patterson AFB, $\mathrm{OH}$
}

\begin{abstract}
Flow control to prevent or delay boundary layer separation can dramatically improve the performance of air vehicles in critical regions of the flight envelope. Prior aerodynamic experiments have shown that unsteady excitation, at the appropriate frequency, can delay boundary layer separation more effectively than steady flow perturbations. An electromechanical flow control device, with a substantial deployment frequency bandwidth, has been developed and tested in both static and dynamic flow control environments. The device couples a high frequency piezostack actuator with a sixty five-to-one displacement amplification mechanism to oscillate sixteen vortex generating blades at rates up to two hundred hertz. Conceptual design of the piezostack-compliant mechanism is discussed. Flow separation control results are presented and device performance issues including dynamic characteristics are addressed.
\end{abstract}

\section{Nomenclature}

$$
\begin{array}{ll}
\text { AOA } & =\text { Angle of Attack } \\
C_{l} & =\text { Lift coefficient, } \frac{L}{S \frac{1}{2} \rho U^{2}} \\
C_{p} & =\text { Pressure coefficient, } \frac{p-p_{\infty}}{\frac{1}{2} \rho U^{2}} \\
f & =\text { unsteady flow disturbance frequency } \\
F^{+} & =\text {reduced frequency (non-dimensional), } \frac{f \cdot X_{t e}}{U} \\
U & =\text { freestream velocity } \\
X_{t e} & =\text { distance from actuator position to trailing edge of flap } \\
\text { HiMVG } & =\text { high frequency micro vortex generator } \\
\text { VG } & =\text { vortex generator }
\end{array}
$$

\footnotetext{
* Senior Aerospace Engineer, Air Force Research Laboratory, AFRL/VAAA, Bldg. 45, $21308^{\text {th }}$ Street, WrightPatterson AFB, OH 45433-7542, Associate Fellow AIAA.

President, FlexSys Inc., 2000 Hogback Road, Suite \#9, Ann Arbor, MI 48105.

\# Vice President, FlexSys Inc., 2000 Hogback Road, Suite \#9, Ann Arbor, MI 48105.

$\S$ Research Scientist \& Lecturer, Univ. of Michigan Aerospace Eng. Dept., FXB building, North Campus, Room 3032, Ann Arbor, MI 48109-2125, Member AIAA.

II Senior Aerospace Engineer, Air Force Research Laboratory, AFRL/VAAA, Bldg. 45, $21308^{\text {th }}$ Street, WrightPatterson AFB, OH 45433-7542, Associate Fellow AIAA.
} 


\section{Introduction}

Flow Control to avoid or delay flow separation can have a major impact on future air vehicle performance particularly if the concept developed is efficient across the speed spectrum. Prior research by FlexSys Inc. ${ }^{1}$ has shown that a high frequency deployable micro vortex generator system (HiMVG) can produce an oscillatory flow field, that when tuned to the boundary layer, is more effective at promoting flow attachment than a static vortex generator array. This finding is consistent with research results published by Wygnanski ${ }^{2}$ and McManus $^{3}$ for oscillatory pneumatic systems. While the precise mechanism of unsteady flow excitation for separation control is not fully understood, the concept involves low level periodic forcing to modulate the formation of vortices in a separating flow. At or near an optimum frequency of excitation, high streamwise vorticity is driven towards the surface delaying separation. This unsteady flow enhancement effect is illustrated in Figure 1. The figure compares the pressure distribution on a deflected trailing edge flap behind both a static and oscillatory vortex generator array. The issue of frequency dependence will be addressed in a subsequence section of the paper.

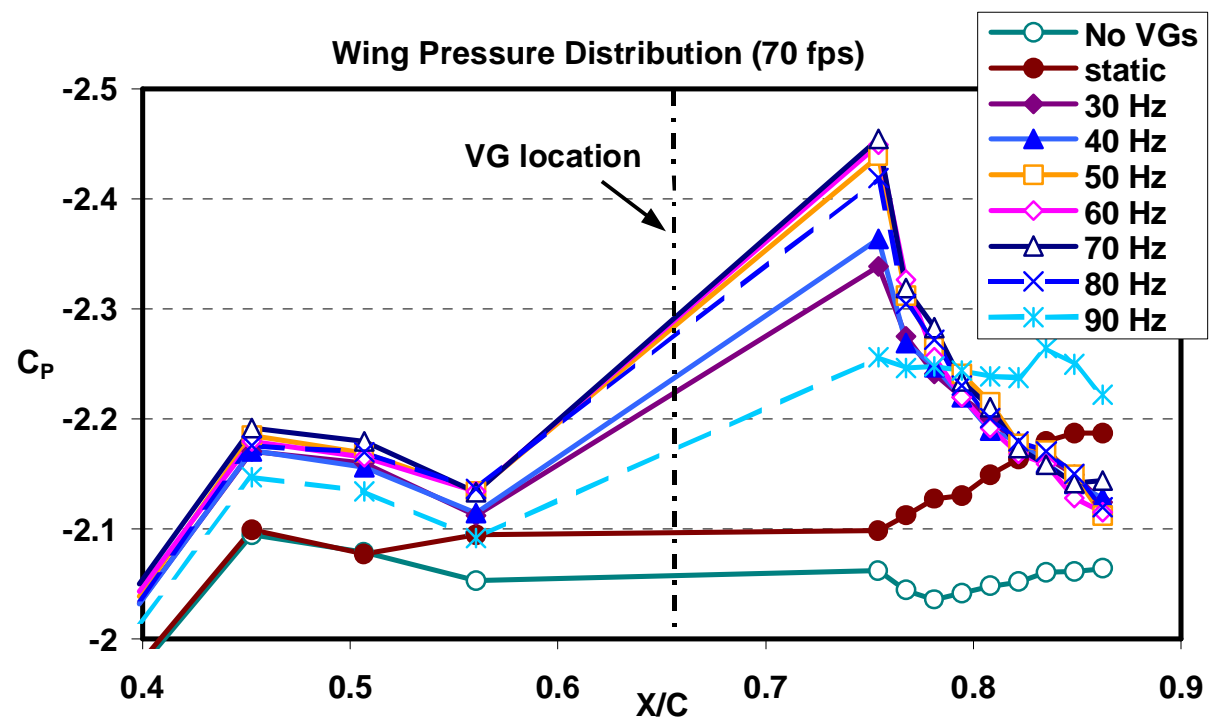

Figure 1: The flow field on a deflected flap with and without active flow control.

The oscillatory flow control results shown were produced with a first generation electro-mechanical device. A 20 to 1 displacement amplification compliant structure designed by Kota, using technology developed at the University of Michigan and extended at FlexSys ${ }^{4}$ is the mechanical heart of the system. The displacement amplification compliant structure shown in Figure 2 amplified the stroke of a voice coil actuator to produce the micro vortex generator deployment height required for effective unsteady excitation of the boundary layer. The vortex generator deployment height could be controlled with consistency across the frequency range of interest.

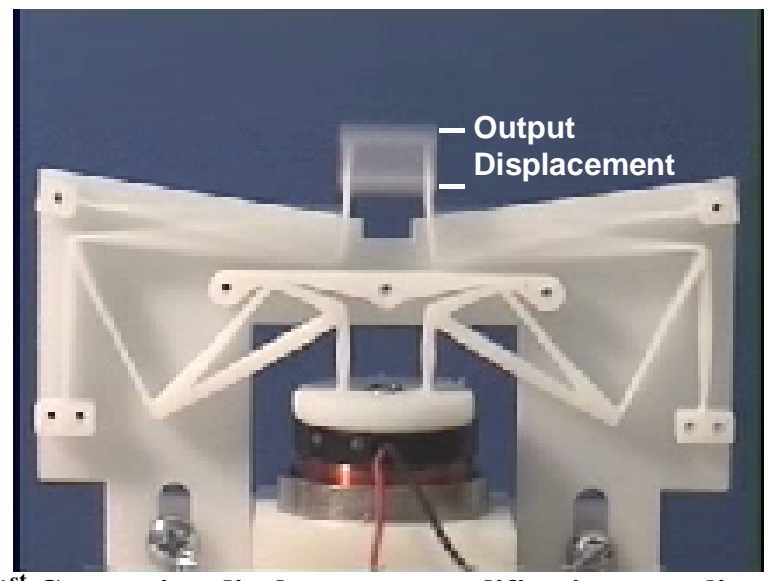

Figure 2: $1^{\text {st }}$ Generation displacement amplification compliant structure. 
The first generation electro-mechanical flow control device proved to be an attractive alternate to the synthetic jet oscillatory flow control devices currently under study. Replacing the voice coil with a piezostack actuator and redesigning the compliant mechanism for better efficiency should produce a system that is competitive with synthetic jets for subsonic flow control applications, and exceeds the synthetic jet in high speed flow separation control environments because of an ability to produce the requisite boundary layer excitation at a low power input. The development and testing of this efficient electro-mechanical device, for active flow control applications follows.

\section{Boundary Layer Separation Control}

To improve the ability of the boundary layer to overcome an adverse pressure gradient, i.e. avoid separation, techniques have been developed to enhance the mixing between the low momentum fluid near the wall and the external high momentum flow. Benchmark flow control experiments by Wygnaski showed that this mixing process becomes extremely efficient if the forcing frequencies correspond to the most unstable frequencies of the separating shear layer downstream of the flow perturbation device. Subsequent boundary layer research ${ }^{3}$ has shown that the nature of the device used to generate the disturbance is not important. In fact, generating unsteady boundary layer disturbances with high frequency mechanical devices dates back to Viets ${ }^{5}$ in the early 1980's. Viets and his colleagues used rotating disks, with appendages that extended beyond the flow surface. The frequency of disturbance was controlled by the rotating disk speed, and to a lesser extent the number of disk tabs.

Most of the modern work with oscillatory flow control has been focused on pneumatic system. The McManus pulsed vortex generator jet concept ${ }^{3}$ is one example. With the McManus system, the vortical flow field is produced by a jet pitched and skewed to the direction of flow. The frequency of this system is controlled by interrupting the air jet before it exits the flow surface. McManus' experiments show a distinct flow separation control advantage when the frequency of the pulsed jet is tuned to the local flow environment. Unfortunately, the pulsed jet system requires a mass flow rate that increases, with the square of the external velocity, to retain its separation control effectiveness. This limits the concepts practical application to the low subsonic speed regime.

On the mechanical device front, $\mathrm{Lin}^{6}$, in his recent work investigating the control of turbulent boundary layer separation, has shown the micro vortex generators if properly sized for the local flow environment can produce strong streamwise embedded vortex structures. Lin scale vortex generators are compatible with piezostack actuator output if a suitable displacement amplification compliant structure can be developed. Lin's research, using static vortex generators, indicated the most effective separation control occurs when the vortex blade induced vortical flow is generated in the region between 0.2 and 0.4 times the boundary layer depth. The Lin aerodynamic design parameters worked effectively for the first generation HiMVG design. Recent measurement of the flow pattern behind a pair of oscillating vortex generators ${ }^{7}$ supports Lin's finding of height effectiveness, and gives insight into how the vortices are switched off and on during vortex generator blade deployment and retraction. The Lin vortex generator blade height parameters, shown in Figure 3, were used to establish displacement amplification criteria for the compliant structure mechanical design.

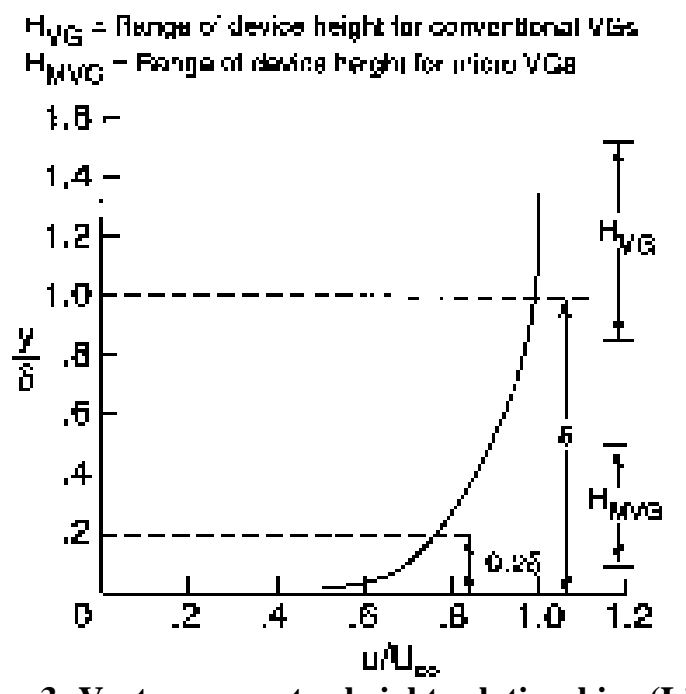

Figure 3: Vortex generator height relationships (Lin). 


\section{Displacement Amplification Compliant Structures}

A compliant mechanism is a class of mechanism that relies on elastic deformation of its constituent elements to transmit motion and/or force. These are in fact mechanisms without joints - neither conventional hinges nor lumped flexural hinges. The mechanisms have compliance distributed throughout the structure and are much more fatigue resistant and easier to manufacture ${ }^{8}$. Distributed compliant systems derive their flexibility due to the topology and shape of the material continuum rather than concentrated flexion at few regions as in plastic hinges.

One of the major barriers in smart structures technology is the displacement, or stroke, available from smart material actuators. In this project, a displacement amplification compliant structure is used as the mechanical heart of the HiMVG active flow control system. Augmenting actuators such as piezoelectric or voice coil actuators with compliant mechanisms leads to systems with actuation functionality built into the structure. Such structures distribute the actuation energy derived from an actuator to the application surface and can efficiently trade force for displacement to obtain the motion requirements for active flow systems. Figure 4 illustrates this type of motion amplification, compliant structure used to drive the high frequency, deployable micro vortex generator. A voice coil motor was used in the first phase of the program to generate motion in the correct frequency range. A piezostack actuator is used to drive the current HiMVG device. The figure below illustrates a first generation piezo-amplifier design that can transform $40 \mu \mathrm{m}$ of input deflection to generate up to $2 \mathrm{~mm}$ of output deflection at frequencies up to $400 \mathrm{~Hz}$.

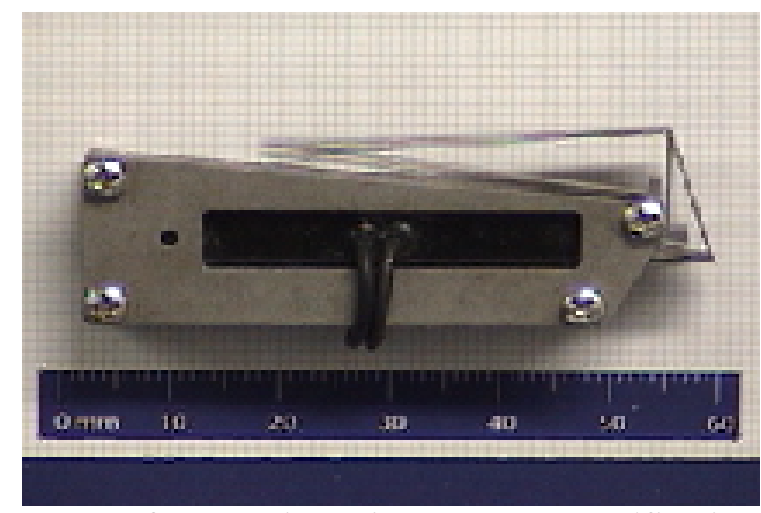

Figure 4: Example of a compliant displacement-amplification mechanism.

The compliant structure is designed to operate over a large working bandwidth below its natural resonance frequency. Design of the HiMVG device used for the phase II aerodynamic investigation will be discussed in the next section.

\section{High Frequency, Deployable Micro Vortex Generator Device}

\section{A. Aerodynamic Design}

The HiMVG development/validation program was structured to investigate flow control on a pitching airfoil. This necessitated locating the deployable vortex generators near the leading edge on the wing upper surface. For the model scale used, the boundary layer is thin at this point and only a $3 \mathrm{~mm}$ deployment height was required for the blade to reach its optimum working height. A trip strip was located ahead of the vortex generator position to insure the boundary would transition from laminar to turbulent flow. The blades were spaced in a co-rotating pattern one inch apart, and at 30 degrees angle-of-attack to the mean flow direction. Figure 5 depicts the micro vortex generator array installed in the dynamic test model. 


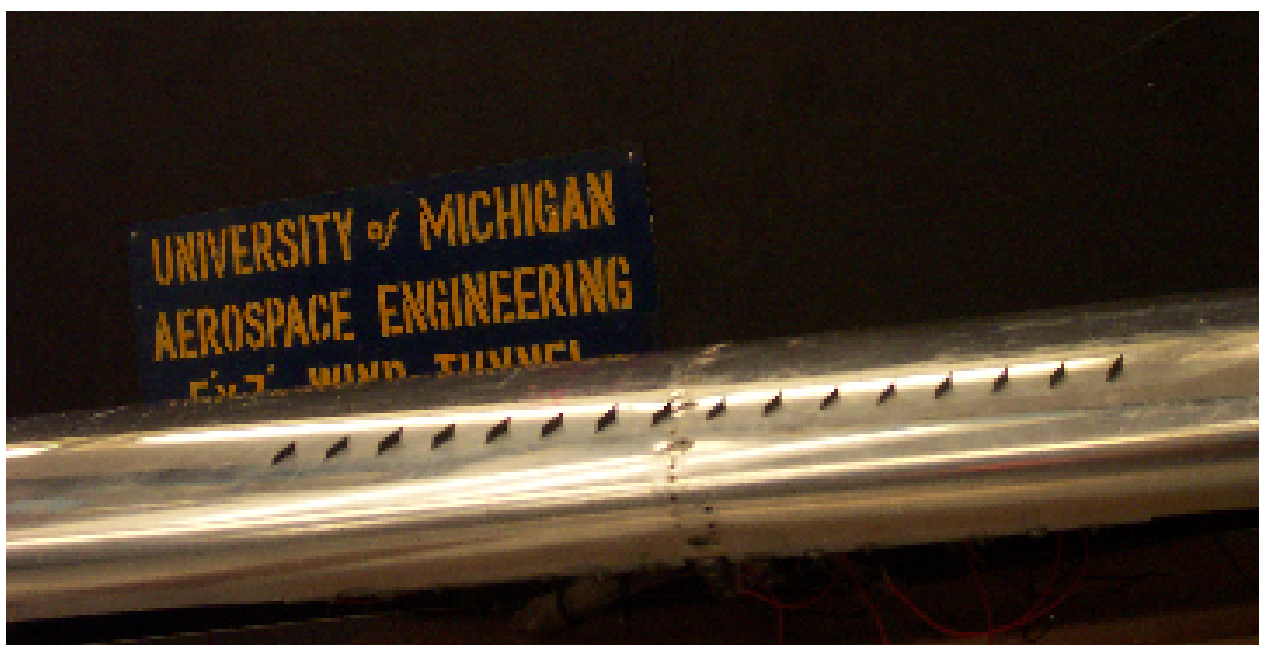

Figure 5: Vortex generator array installed in the pitching wing model.

The individual blades were configured in a trapazodial shape, as shown, with the end of the blade contoured to match the airfoil upper surface when the blades are retracted. A static test model, with stationary blades installed, was tested in the wind tunnel to verify blade position and height before the actual displacement amplification compliant structure design activity was initiated.

\section{B. Compliant Structure Design}

Using in-house compliant structures design tools, a displacement amplification compliant structure was optimized for the micro VG system. Compliant structures (or compliant mechanisms) are advantageous in that they can work with very small deflections generated by electrostrictive, or other types of smart materials actuators and trade force for deflection - much like a simple lever ${ }^{9}$. Figure 6 illustrates a compliant structures ability to alter the force-displacement characteristics of smart materials actuators.

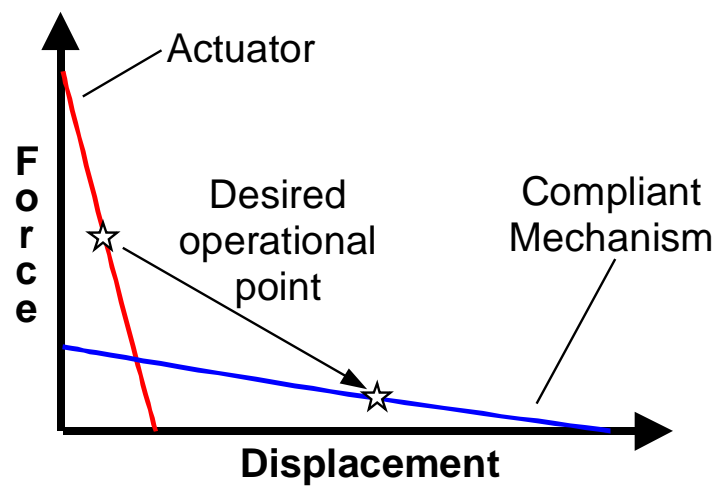

Figure 6: Actuator-tailoring using compliant mechanisms.

The advantage of the piezoelectric stack actuator is that they possess a high power density and can operate at high frequencies. Piezoelectric actuators also can produce large forces; however, they do not produce sizable deflections unless bender or mechanical amplifier technologies are utilized. The primary disadvantage of bender configurations are the resulting stiffness to mass ratio of the device such the first natural frequency is typically lower than $100 \mathrm{~Hz}$. Since the active flow system needed to operate at frequencies exceeding $100 \mathrm{~Hz}(300 \mathrm{~Hz}$ was the target frequency), bender type configurations could not be used.

The compliant structure was configured to take the force/displacement characteristics an APC piezoelectric stack actuator (Dimensions: 5x5x36 mm). The low voltage stacks require 150 Volts for maximum performance and can produce a maximum block force of $750 \mathrm{~N}$ and a free deflection of $60 \mu \mathrm{m}$ with a capacitance of $3600 \mathrm{nF}$. These characteristics were placed into a structural optimization algorithm that was designed to maximize the free deflection and block force of the actuator of the compliant mechanism system (natural frequency is indirectly maximized). The optimization algorithm also included a constraint on the maximum stress in the structure and a 
constraint on the geometric advantage (inverse of mechanical advantage) that controlled the size of displacement amplification obtained by the mechanism. Thus, the algorithm maximizes the mechanical efficiency of the compliant amplifier at the specified geometric advantage and stress limit. The compliant structure was investigated with a variety of materials including, steel, titanium, and aluminum. Optimization started with two-dimensional beam element models. Once a preliminary design was chosen, further shape refinement was performed using twodimensional plane strain models to model the detailed stress field in the structure (stress concentrations due to fillets).

The finalized design was fabricated using wire EDM technology from titanium. Some additional modeling was done to assess the dynamic impact of the mass of the vortex generator system. The final design could produce a maximum deflection of $2.97 \mathrm{~mm}$ and a maximum block force of $4.1 \mathrm{~N}$. The first natural frequency of the combined actuator-compliant amplifier was $300 \mathrm{~Hz}$. Figure 7 illustrates an array of eight actuator-amplifiers with a total of 16 vortex generators blades. Adding the mass of the blades reduced the first natural frequency down to $180 \mathrm{~Hz}$ due to the blade and bar to couple two blades together. Higher frequency actuators could be achieved by using an individual blade per actuator-amplifier which pushed the $1^{\text {st }}$ natural frequency to approximately $240 \mathrm{~Hz}$.

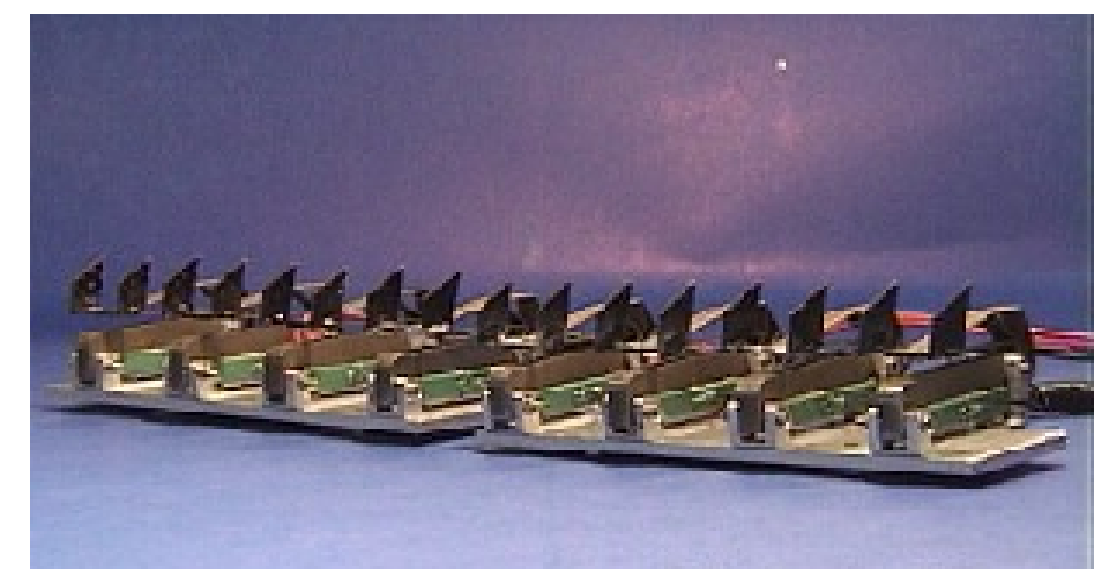

Figure 7: Eight actuator-amplifier array with 16 vortex generator blades.

\section{Boundary Layer Separation Control Aerodynamic Testing}

Primary flow control testing for this program was conducted in the University of Michigan 5' x 7' subsonic wind tunnel. The wing model used during the testing program had a chord length of two feet and a span of four feet. The test airfoil section was the PE 1423, a modern trailing edge separation airfoil that had been tested previously ${ }^{7}$. Figure 8 shows the wing model mounted in the wind tunnel ready for testing. The active vortex generator array, sixteen blades total, spanned the centerline of the model. At both ends of the active array eight static vortex generators were positioned to minimize contamination in the test area as the wing approached stall. This technique proved effective during trailing edge flap separation control testing ${ }^{1}$. The model was instrumented with a chordwise row of static pressure taps located at the centerline. These pressures were integrated to determine model lift as a function of angle-of-attack.

\section{A. Static Flow Control Experiments}

HiMVG device performance was first determined with the model positioned at a series of static angles-ofattack. The purpose of this initial test series was to determine device turbulent boundary layer separation control performance as a function of device deployment frequency, and compare these results with the aerodynamic data base established during phase one of the program. Phase one program results ${ }^{1}$ demonstrated conclusively that the boundary layer was better strengthened when the device deployment was such that the reduced frequency of the system, $\mathrm{F}^{+}$, was approximately equal to one $\left(\mathrm{F}^{+}=f^{*} \mathrm{X}_{\mathrm{te}} / \mathrm{U}\right)$. The static lift performance shown in Figure 9 was taken at a freestream velocity, $\mathrm{U}=132 \mathrm{ft} / \mathrm{sec}$. This velocity and the geometric characteristics of the model yield an $\mathrm{F}^{+}$ equal to one if the devices are oscillated $70 \mathrm{~Hz}$. Figure 9 shows the "best" separation control occurs at this frequency. The next illustration, Figure 10, presents a comparison of performance between static vortex generators extended to 0.2 (VGL) and 0.4 (VGH) times the boundary layer thickness and an oscillating VG array operating at $70 \mathrm{~Hz}$. The oscillating VG extended to 0.4 delta and retracted to the surface of the airfoil. The oscillating array 
improved the maximum lift of the airfoil $9 \%$ and increased the maximum lift angle-of-attack by approximately 1.5 degrees.

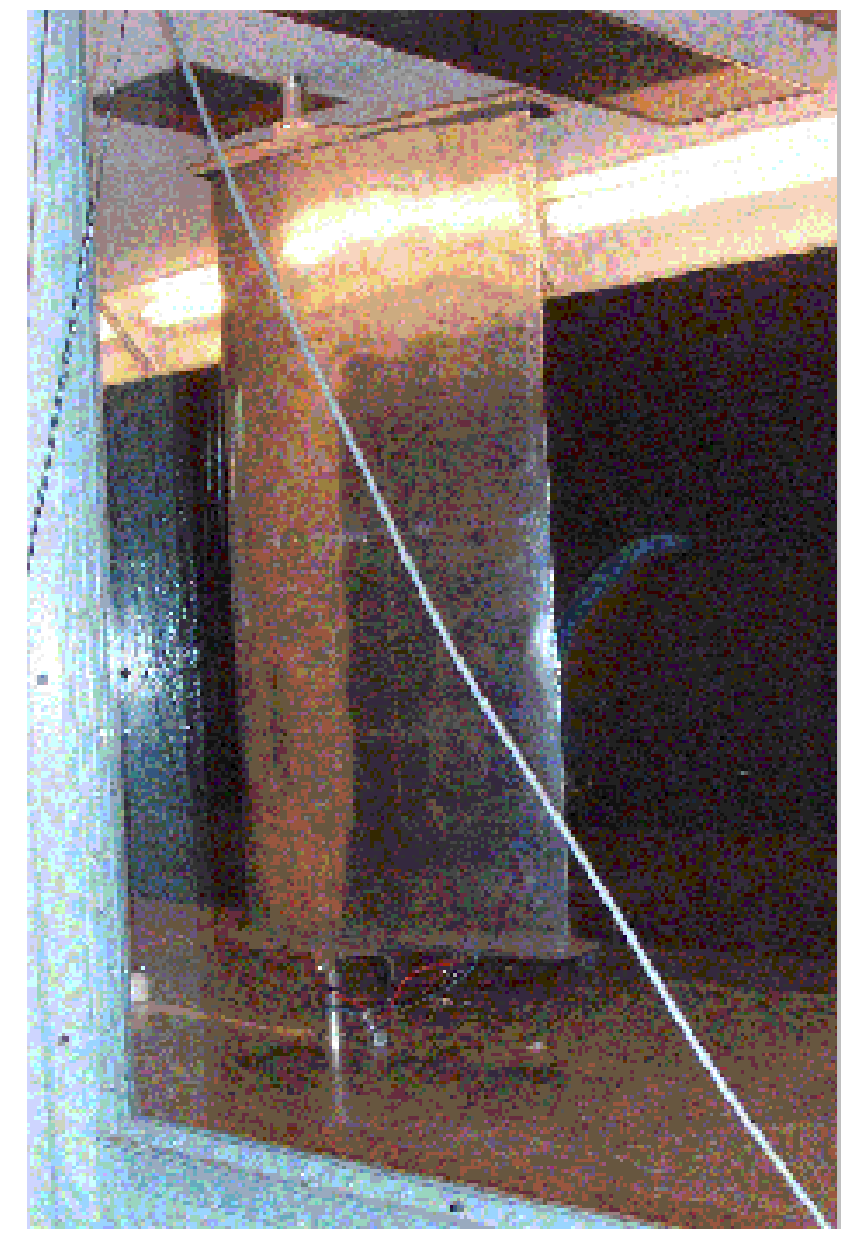

Figure 8: Instrumented test wing installed in the $\mathrm{U}$ of $\mathrm{M}$ subsonic wind tunnel.

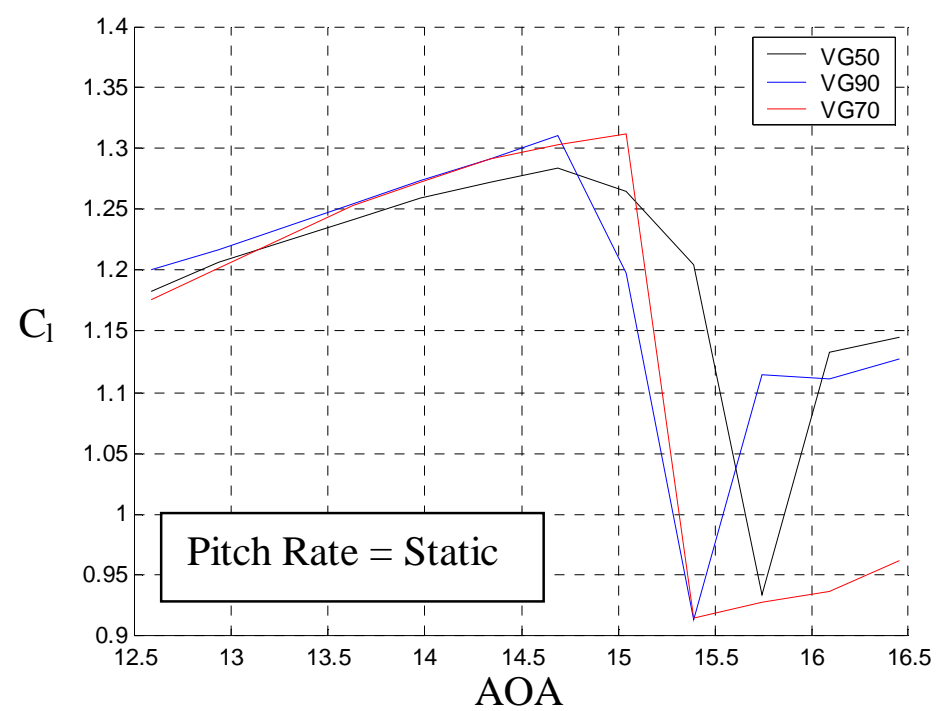

Figure 9: Lift as a function of HiMVG deployment frequency; $\mathrm{U}=132 \mathrm{ft} / \mathrm{sec}$. 


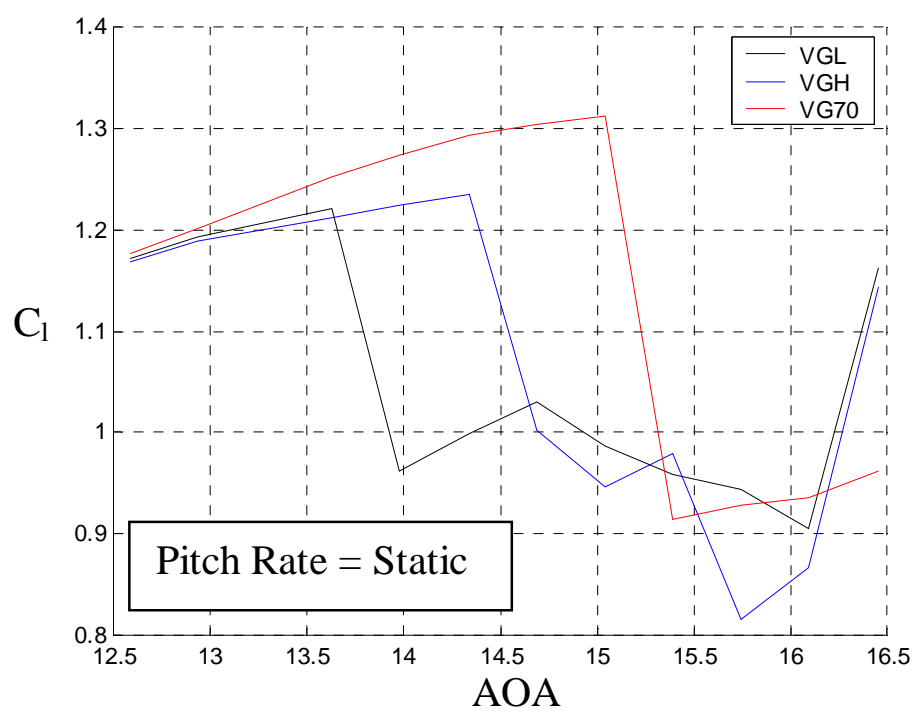

Figure 10: Static and dynamic vortex generator lift comparison; $U=132 \mathrm{ft} / \mathrm{sec}$.

The static model test results make two important points; first, that oscillatory flow effectors are "better" than static devices at strengthening the boundary layer, and second, in a subsonic flow environment producing a flow control disturbance at a reduced frequency of one enhances boundary layer attachment more than other disturbance frequencies. This is consistent with the findings of other researchers working in the area of oscillatory flow control.

\section{B. Dynamic Flow Control Experiments}

The dynamic flow control aerodynamic tests were conducted at three model pitch rates 7.5, 15, and 22.5 degrees per second. Pressures were taken with high response rate PSI units. Only one pressure was recorded during each pitch sweep, and all the lines from the pressure tap to the recording module were the same length. This technique has been used successfully in the past to collect dynamic pressure data on pitching wind turbine blade sections. The VG's in the deployed position extended $3 \mathrm{~mm}$ beyond the surface which was approximately 0.4 times the boundary layer thickness at that point on the wing chord. A trip was used ahead of the VG array to insure the boundary layer was turbulent at the active control point.

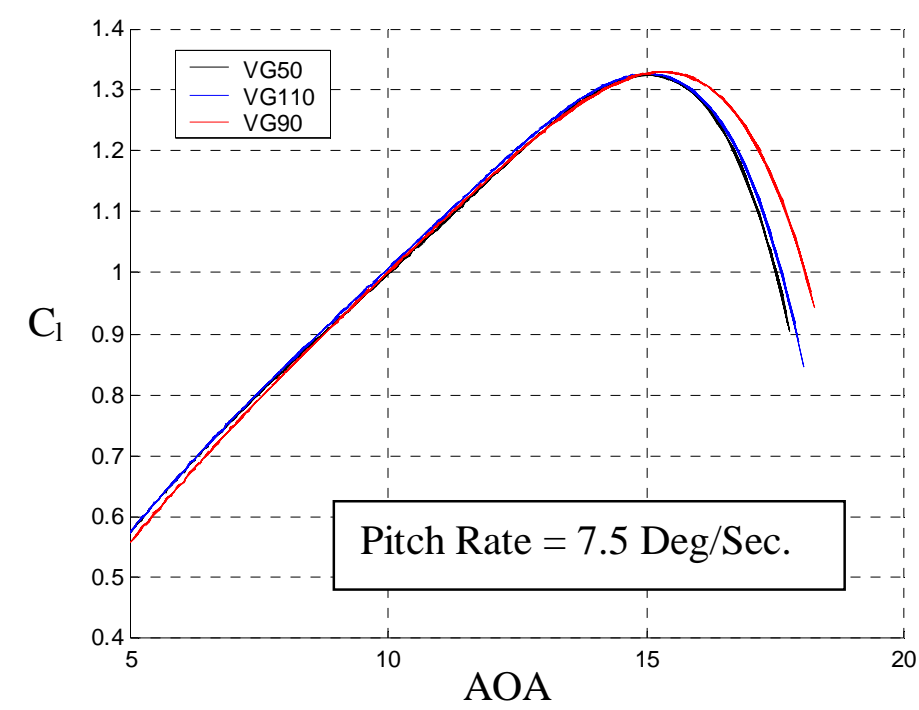

Figure 11a : Static versus dynamic flow control on a pitching wing; $U=132 \mathrm{ft} / \mathrm{sec}$. 


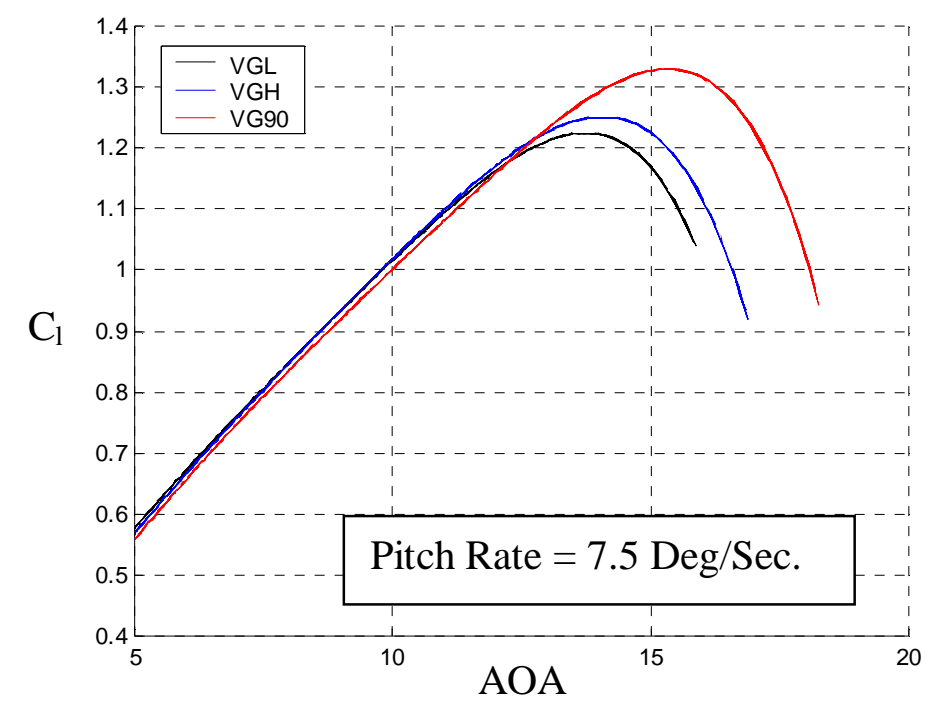

Figure 11b : Active flow control frequency trend on a pitching wing; $\mathrm{U}=132 \mathrm{ft} / \mathrm{sec}$.

The HiMVG devices produced additional lift when compared to static VG blades at the three frequencies tested (Figure 11a and 11b). However, the additional lift (better flow attachment) did not exhibit the $\mathrm{F}^{+}=1$ frequency maximums observed in the static data (Figure 12) at the higher pitch rates. Also, as the pitch rate increased the leading edge shed vortex began to dominate the wing pressure field and the lift increments due to oscillatory flow control were lost in the global flow field effects.

For the pitching wing, optimal HiMVG oscillatory frequencies $\left(\mathrm{F}^{+}=1\right)$ correlate to static and low pitch rate results. Lower frequency device oscillations seem to work better at higher pitch rates. However, global flow field events cloud this issue, and more study is required.

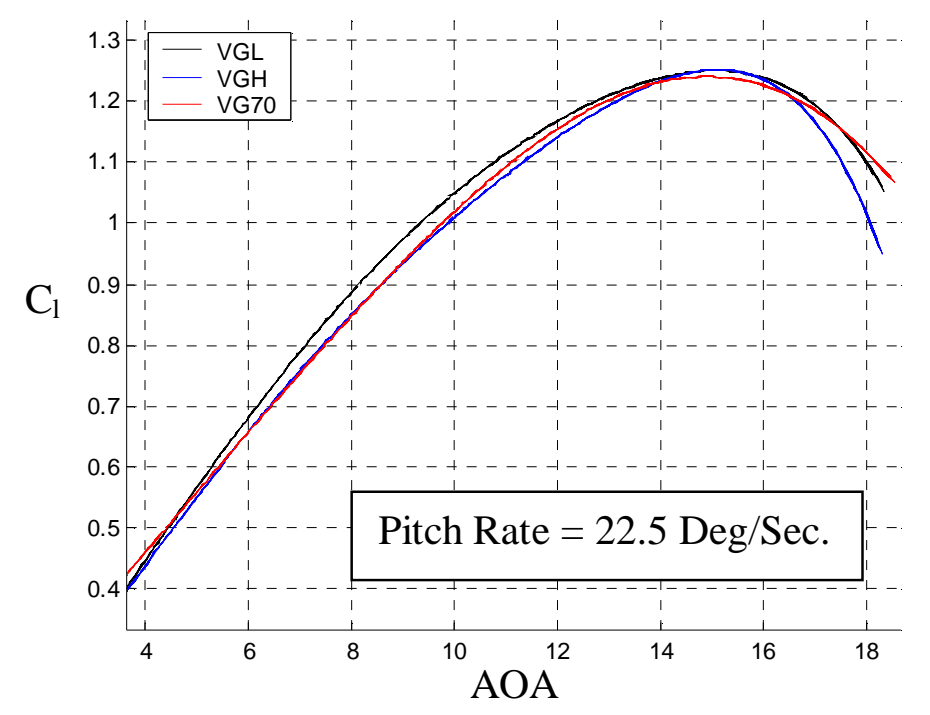

Figure 12 : Global dynamic lift increment; $U=132 \mathrm{ft} / \mathrm{sec}$.

\section{High Speed flow Control}

Near the end of the program an opportunity presented itself to quickly evaluate HiMVG performance in a separating transonic flow environment. Since a claimed primary advantage of the electro-mechanical device developed during this program is that it requires no additional power to affect a separating boundary layer at speeds higher than subsonic, the opportunity to demonstrate its performance was eagerly accepted. Testing was conducted in the Notre Dame 4" x 4" transonic wind tunnel located on the university campus. 
The test set-up was similar to the one used to study cavity shear layer control. Figure 13 is a photograph of the in-draft wind tunnel with the FlexSys HiMVG system installed in the test section $30 \mathrm{~mm}$ upstream from a 20 degree pressure instrumented ramp. The HiMVG devices used in the transonic experiment were the same ones used in the static and dynamic subsonic flow control experiments discussed previously, with one modification. Each displacement amplification device drove a single vortex generator blade instead of the two used in the subsonic experiments. This modification was made to increase the deployment frequency band.

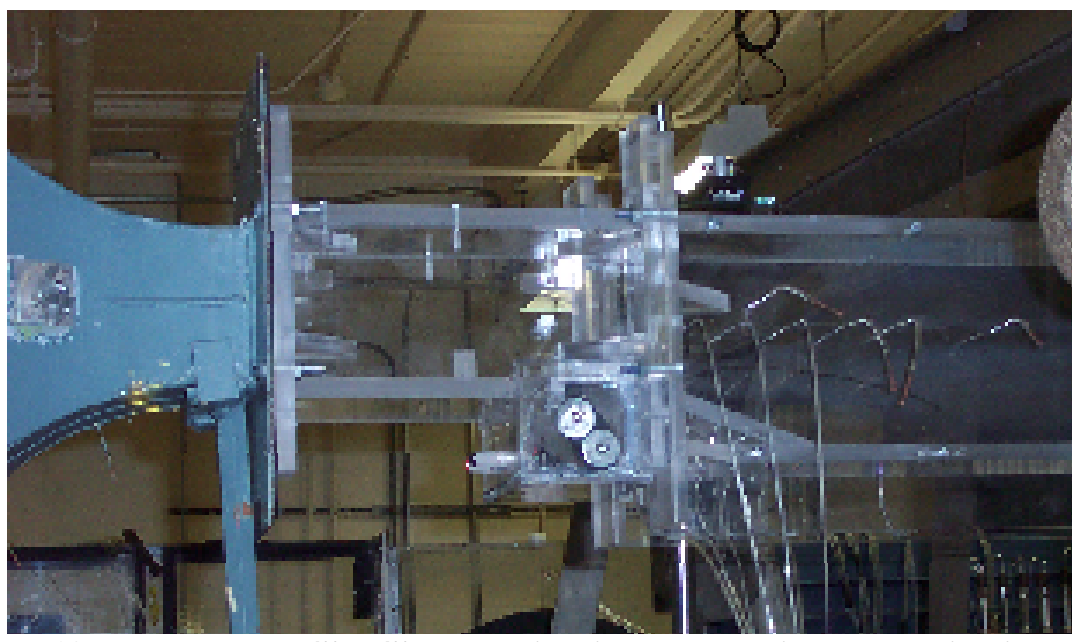

Figure 13 : Notre Dame 4" x 4" transonic wind tunnel with test section installed.

The next figure is a close-up view of the HiMVG high frequency, deployable micro vortex generator devices and the test section installation. The array dimensions were the same as the one used in the subsonic experiment. However, the deployment height was adjusted downward slightly from the $3 \mathrm{~mm}$ used in the subsonic experiments after measuring tunnel floor boundary layer thickness at the VG location. The maximum deployment height of the blades during testing was 0.4 times the boundary layer thickness at the VG location.

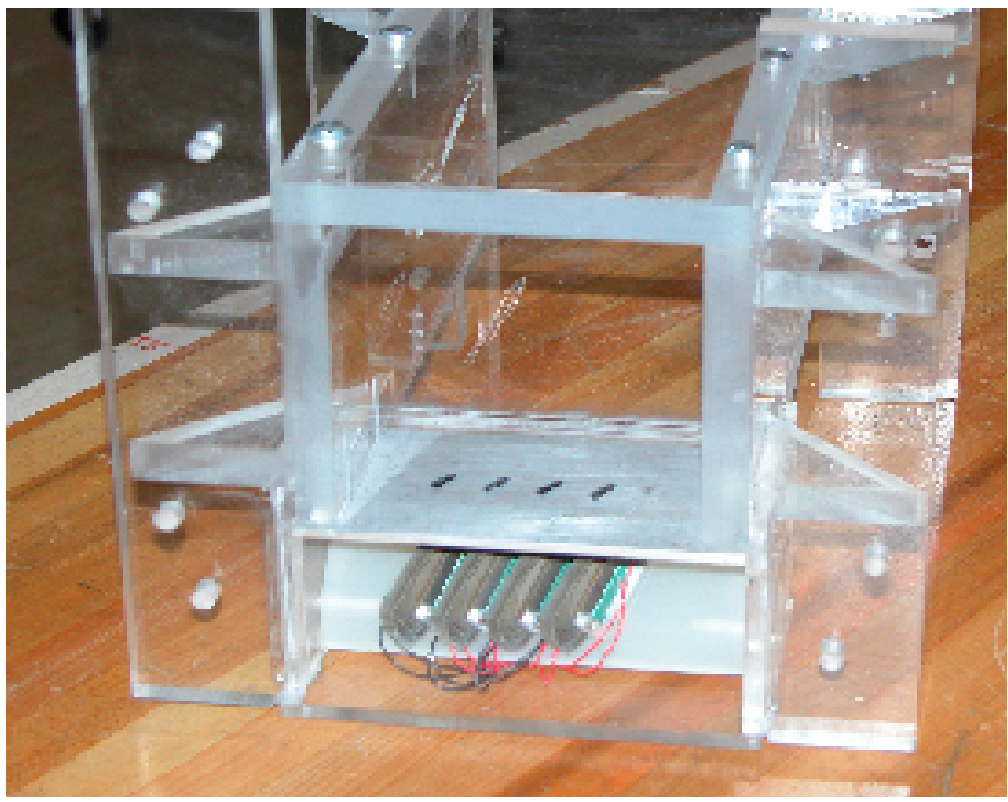

Figure 14 : HiMVG devices installed in the test section.

Initial flow control testing was conducted at two Mach numbers: 0.32 and 0.52 . Testing at higher transonic velocities was aborted when a tunnel wall flow separation problem occurred that could not be corrected during the short two-day test period. However, information gleaned from the test indicates the HiMVG device, with further 
development, has the potential for being effective at controlling flow separation in a transonic environment. The array shown effectively turned and attached the flow on the 20-degree ramp surface at both test Mach numbers. Tuft visualization on the ramp picked up the pulsing flow pattern generated by the oscillating blades. Pressure measurements on the ramp were affected by sidewall separation and could not be analyzed to determine shed vortex frequency effect on the extent of flow attachment.

\section{Future Device Development}

The maturation process for the HiMVG device needs to proceed in three directions. First, and the most near term, is modifying and testing the existing device in a transonic flow separation control environment of major interest to the aerospace industry. A good example application might be an aggressive natural laminar flow airfoil design that requires boundary layer energy addition, at the beginning of the upper surface pressure recovery region, to prevent massive flow separation during aircraft maneuvers. Recent performance studies ${ }^{10}$ suggest that long endurance aircraft can benefit significantly if the run of laminar flow on the airfoil upper surface can be increased from 55 to $70 \%$ of the chord length.

A second and more difficult device development would be the design, fabrication and test of a displacement amplification compliant structure with variable resonance. Compliant devices like the HiMVG require the least power when the structure is operating near resonance. On the other hand many aerospace applications require that flow disturbances be generated across a broad frequency band. Tuning the structural resonance of the system to match the frequency output could reduce the power required by an order of magnitude if the operating frequency range is large.

The third future HiMVG device development being considered has ramifications that could lead to the discovery of a practical scheme for turbulent flow drag reduction. The device being considered is MEMS size, and several samples have been designed fabricated and tested for other applications. Figure 15, shows an example of an electro-static actuator driving a compliant amplifier at $27 \mathrm{KHz}$. The compliant mechanism was designed by Kota and fabricated at Sandia National Labs ${ }^{11}$. It is postulated that a similar size mechanism, driving vortex shedding pins into the sub-layer of a turbulent boundary layer, can smooth out the scrubbing vortex structures that are a major source of turbulent skin friction drag. Koumoutsakos ${ }^{12}$ gives a good explanation of how to actively control vortex wall interactions for drag reduction. Adaptation of a compliant mechanism to produce the control disturbances identified at the correct frequency may turn into a complex research project, but the potential drag reduction reward could be well worth the basis research effort involved.

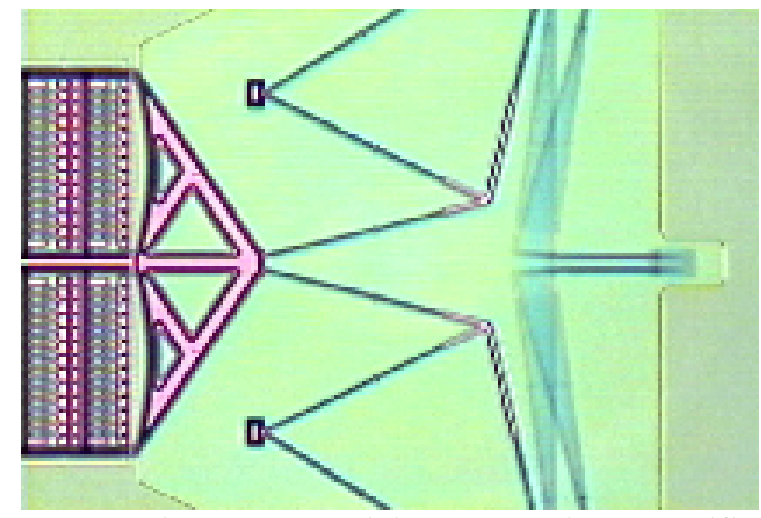

Figure 15 : Electro-static actuator driving a compliant amplifier at $26.9 \mathrm{KHz}$.

\section{Conclusions}

This research effort made significant strides in the development and demonstration of an electro-mechanical device for oscillatory flow control applications.

- A compact piezostack driven displacement amplification structure that deployed micro vortex generator blades into a turbulent boundary layer at frequencies up to $240 \mathrm{~Hz}$ was developed and tested in several separating turbulent boundary layer environments.

- The flow attachment performance at subsonic test velocities matched that of the best oscillatory pneumatic devices during static model testing. 
- Dynamic flow control on a pitching wing was less conclusive. Good flow attachment results were achieved at low pitch rates. Device effect on the global flow field at high pitch rates was marginal.

- The same HiMVG devices used during low speed testing were able to attach flow on a 20-degree ramp, at Mach 0.32 and Mach 0.52 freestream conditions, using no more power input than that used in the low speed flow control experiments.

- Power required to operate a single HiMVG device is on the order of one watt when the deployment frequency is near the actuator-amplifier resonance frequency.

\section{Acknowledgments}

The authors would like to acknowledge funding provided for this program by the Air Force Office of Scientific Research (AFOSR), and the support from our Program Manager, Dr. John Schmisseur

\section{References}

${ }^{1}$ Osborn, R., Kota, S., Geister, D., Lee, M., Tilmann, C., "Active Flow Control Using High Frequency Compliant Structures," AIAA Paper 2001-4144, AIAA Atmospheric Flight Mechanics Conference \& Exhibit, Montreal, Canada, August 2001.

${ }^{2}$ Wygnanski, I., "Some New Observations Affecting the Control of Separation by Periodic Excitation," AIAA Paper 2000-2314, 2000.

${ }^{3}$ McManus, K., et al., "Pulsed Vortex Generator Jets for Active Flow Control," AFRL-VA-WP-TR-1998-3028, Nov. 1997.

${ }^{4}$ Kota, S., Hetrick, J., Li, Z., Saggere, L., "Tailoring the Force/Displacement Characteristics of Unconventional Actuators Using Compliant Mechanisms," IEEE/ASME Transactions on Mechatronics, Vol. 4, No. 4, pp 396-408.

${ }^{5}$ Viets, H., Piatt, M., Ball, M., "Forced Vortices Near a Wall," AIAA-81-0256, January 1981.

${ }^{6}$ Lin, J.C., "Control of Turbulent Boundary Layer Separation Using Micro-Vortex Generators," AIAA Paper 993404, $30^{\text {th }}$ AIAA Fluid Dynamics Conference, Norfolk, VA, June 28 - July 1, 1999.

${ }^{7}$ Lisy, F. J., et al., "Smart Surfaces for Flow Control," AFRL-VA-WP-TR-1999-3075, Jan 1999.

${ }^{8}$ Osborn, R., Hetrick, J., Kota, S., Geister, D., Lee, M., and Joo, J., "High Frequency Vortex Generators for Active Flow Control using Compliant Systems," AFOSR TR, dated May 2001, to be published.

${ }^{9}$ Kota, S., Hetrick, J., Osborn, R., Paul, D., Pendleton, E., Flick, P., Tilmann, C., "Design and Application of Compliant Mechanisms for Morphing Aircraft Structures," SPIE-2003-5054-3, SPIE Smart Structures and Materials Conference, 2-6 March 2003.

${ }^{10}$ Tilmann, C., Flick, P., Martin, C., Love, M., "High Altitude Long Endurance Technologies for SensorCraft," RTO AVT "Symposium on "Novel Vehicle Concepts and Emerging Vehicle Technologies," Brussels, Belgium, 710 April 2003.

${ }^{11}$ Kota S., Hetrick, J., Li, Z., Rodgers, S., Krygowski, T., "Synthesing High-Performance Compliant Stroke Amplification Systems for MEMS", Proceedings ofthe IEEE Thirteenth International Micro Electro MechanicalSystems Conference, Miyazaki, Japan, January 23, 2000, pp.164-169.

${ }^{12}$ Koumoutsakos, P., "Active Control of Vortex-Wall Interactions," Phys. Fluids, Vol. 9, No. 12, 1997, pp 3808-3816. 
American Institute of Aeronautics and Astronautics 